\title{
Rebamipide and Pantoprazole Combination in
} NSAIDs-Gastropathy Treatment

\author{
Maher Mbarki $^{1}$, Helen Sklyarova ${ }^{1}$, Krystyna Aksentiychuk $^{1}$, Natalia Kharchenko ${ }^{2}$ and Eugene Sklyarov ${ }^{1}$ \\ 1. Department of Therapy No. 1 and Medical Diagnostics, Lviv National Medical University Named After Danylo Halytsky, Lviv \\ 79010, Ukraine \\ 2. Department of Gastroenterology, Dietology and Endoscopy, Shupyk National Medical Academy of Postgraduate Education, Kyiv \\ 04112, Ukraine
}

\begin{abstract}
The objective of this study was to investigate the pantoprazole and rebamipide efficiency on NSAIDs (nonsteroidal anti-inflammatory drugs)-gastropathy restoring and healing in patients with coronary heart disease, stable angina, who have been taking aspirin for a long time period. The study included three groups of patients according to the treatment they have got: ASA (acetylsalicylic acid) in the first group; ASA and pantoprazole in the second; ASA, pantoprazole and rebamipide in the third one. To obtain the results, endoscopic method and proinflammatory cytokines LTB4 and protective prostaglandin E2 determination in the blood were used. The research demonstrated no ulcer effects in the group of patients who were treated by rebamipide, and significantly fewer gastroduodenal erosions, in comparison to the group, where treatment contained ASA and pantoprazole. The LTB4 (leukotriene B4) level decreased in pantoprazole and rebamipide treatment groups, but the PGE2 (prostaglandin E2) level increased only after rebamipide therapy. Therefore, rebamipide should be included to the therapy for the better NSAIDs-gastropathy treatment, in reason of its good reparative and gastroprotective properties.
\end{abstract}

Key words: NSAIDs, acetylsalicylic acid, pantoprazole, rebamipide, gastropathy, LTB4, PGE2.

\section{Introduction}

NSAIDs (nonsteroidal anti-inflammatory drugs), including ASA, is the cause of gastroduodenal erosive and ulcerative lesions, bleeding and its associated perforation [1]. The main mechanism of the NSAIDs damaging effect on stomach and duodenum mucosa is the COX (enzyme cyclooxygenase blockade), which has two structural isomers (constructive): COX-1 and COX-2 induced. NSAIDs interrupt the cyclooxygenase pathway of arachidonic acid metabolism by inhibiting the synthesis of PG (prostaglandine), prostacyclin and thromboxane [2]. NSAIDs, as weak organic acids, easily penetrate the phospholipid membrane in the cell cytoplasm, causing local damage to the stomach and duodenum mucosa as erosions and ulcers [3]. At the same time, the synthesis of leukotrienes increases

Corresponding author: Eugene Sklyarov, M.D., Prof., research fields: gastroenterology and internal medicine.
(LTB4 and others), inducing stomach mucosa local inflammation and toxic damage. It is accompanied by the active neutrophils adhesion and chemotaxis on epithelial cells, damaged microcirculation in the gastric mucosa lamina propria [4].

The drugs for parenteral and rectal assignment reduces the risk of erosive and ulcerative lesions in the stomach and duodenum mucosa only in the first 7 14 days of treatment, but then systematic prostaglandin synthesis inhibition due the NSAIDs effect starts up, regardless of the preparation form and administration ways [5]. According to Maastricht-4 recommendations, combination therapy with PPIs (proton pump inhibitors), where pantoprazole is one of the major, and antibiotics should be carried out for patients with NSAIDs-gastropathy [6].

Therefore, the inclusion of drugs with gastrocytoprotective effect, in particular rebamipide in the traditional treatment regimens attracted attention 
recently [7]. It is a gastroprotective drug, which has been clinically proven to effectively gastric ulcers healing and prevention NSAIDs [8]. It reduces inflammatory cytokines and chemokines productivity, decelerates neutrophil activation, what decrease gastroduodenal damage and, probably, is carcinogenesis prevention [9].

However, the rebamipide role in treatment regimens of gastroduodenal erosive and ulcerative diseases, associated with NSAIDs, was not studied completely.

The aim of this study was to analyze the effectiveness of pantoprazole and rebamipide combination in NSAIDs-gastropathy treatment in patients with coronary heart disease, stable angina, who have been taking aspirin for a long time period.

\section{Materials and Methods}

The study involved 102 patients (55 males (54\%) and 47 females (46\%) with the average age $64.7 \pm$ 1.08 years) with NSAIDs-gastropathy, combined with coronary heart disease, stable angina which have been taking ASA prophylactically in a dose of $75 \mathrm{mg}$ /day. According to the treatment, three groups of patients were formed. The first group included 37 patients, who received the ASA. The second group (33 patients) — pantoprazole $40 \mathrm{mg}$ /day adding to the ASA. The third group of patients has been treated by $100 \mathrm{mg}$ rebamipide three times a day in addition to pantoprazole and ASA. Depending on the disease anamnesis, patients received ASA from 1 to 7 years (the average length of $2.66 \pm 0.15$ years).

General clinical examination was performed to all patients, which included data of past medical history, laboratory tests, H. pylori stool-test. The endogenous PGE2 (prostaglandin E2) serum content was investigated using ELISA reagent set PGE2 Immunoassay R\&D Systems on control calibration graph building and the received values comparing. LTB4 plasma concentrations were determined using ELISA test kits LTB4 (Neogen corporation, USA) with the schedule construction of the calibration chart using the standard curve.

During EGDS (esophagogastroduodenoscopy), visual assessment of gastroduodenal region mucosa morphological pattern was executed. The concentration was on to the presence of hyperemia, edema, erosive and ulcerative lesions, duodenal bulb scar deformity, cardiac sphincter tone, presence of gastroesophageal reflux.

Statistical analysis included the comparison group which was conducted with the Mann-Whitney, Tau-Kendall coefficient. There were several research groups in this study, so the reliability of difference in patients treated with the different drugs, was determined by Newman-Keuls criterion.

\section{Results and Analysis}

It was investigated the gastric mucosa and duodenal statement by the endoscopy method in three groups of patients depending on the choice of treatment. The results are shown in Table 1.

According to Table 1, in 6 patients of ASA group, ulcerative defects in stomach were noticed, which was three times more in comparing with patients, who received PPI (pantoprazole). In contrast, in patients who took pantoprazole and rebamipide in addition to ASA, ulcers were not observed.

Table 1 Endoscopic mucosal changes of gastroduodenal zone in patients with cardiovascular disease according to the prescribed treatment.

\begin{tabular}{lllllll}
\hline Endoscopic changes & $\begin{array}{l}\text { Group 1 } \\
(\mathrm{ASA}, n=37)\end{array}$ & $\begin{array}{l}\text { Group 2 } \\
(\text { ASA + PPI, } n=33)\end{array}$ & $\begin{array}{l}\text { Group 3 } \\
(\text { ASA + PPI }+ \\
\text { Rebamipide, } n=32)\end{array}$ & $p(1 \sim 2)$ & $p(1 \sim 3)$ & $p(2 \sim 3)$ \\
\hline Gastric ulcers & $6(6 \%)$ & $2(2 \%)$ & 0 & $>0.1$ & 0.024 & $>0.1$ \\
Gastric erosions & $28(26 \%)$ & $14(14 \%)$ & $7(7 \%)$ & $>0.1$ & 0.002 & $>0.1$ \\
Duodenal ulcers & $4(3.9 \%)$ & $2(2 \%)$ & 0 & $>0.1$ & $>0.1$ & $>0.1$ \\
Duodenal erosions & $19(19 \%)$ & $8(7.8 \%)$ & $4(3.9 \%)$ & $>0.1$ & 0.026 & $>0.1$ \\
\hline
\end{tabular}

$p$ : significance value. 
Duodenal ulcerative lesions were found in 4 patients from group which took the ASA. That was twice as much as in the group of pantoprazole and ASA treatment. In group of therapy by rebamipide, duodenal ulcers were not found.

As for stomach erosive defects, the highest percent was detected in patients who were getting ASA, and the smallest one in the group where the treatment was accompanied by rebamipide adding. 14 erosive lesions were found in the group of patients, who have been treated by pantoprazole and ASA, which was twice less than in the ASA group and higher twice in comparing to the rebamipide group.

Duodenal erosions were found in 19 patients of ASA group, while almost 5 times less analogically defects were observed in patients who were treated by rebamipide and pantoprazole along. Number of duodenal erosive defects in group of pantoprazole therapy equal to 8 , which was significantly lower comparing to ASA and much more than rebamipide groups.

Thus, pantoprazole and rebamipide adding to ASA therapy significantly reduced the number of gastric ulcers $(p<0.05)$, gastric erosions $(p<0.01)$, duodenal erosions $(p<0.05)$ in patients with NSAIDs gastropathy.

To show clearly the rebamipide and pantoprazole effectiveness in NSAIDs-gastropathy treatment, inflammatory cytokines LTB4 were measured (Fig. 1).

The highest LTB4 level $(50 \mathrm{ng} / \mathrm{mL})$ was in patients who took ASA. More than twice its level was lower in patients, who were treated by pantoprazole. Also, in comparison to the ASA group, the level of LTB4 was low in group, which was treated by rebamipide, but not significantly different from the group of pantoprazole therapy. Although the changes between the groups were not significant, but downward trend of proinflammatory mediators in the group, where the part of treatment was rebamipide, have been marked.

Prostaglandins, which is an important marker of gastroduodenal mucosa defects healing and regeneration, were measured for three groups of patients to compare the pantoprazole and rebamipide reparative effect on NSAIDs gastropathy (Fig. 2).

The level of PGE2 in patients, which were taking the ASA, was slightly higher, in contrast to the group, where treatment included pantoprazole. However, in patients, who were getting rebamipide, it was significantly higher than its rates in both groups.

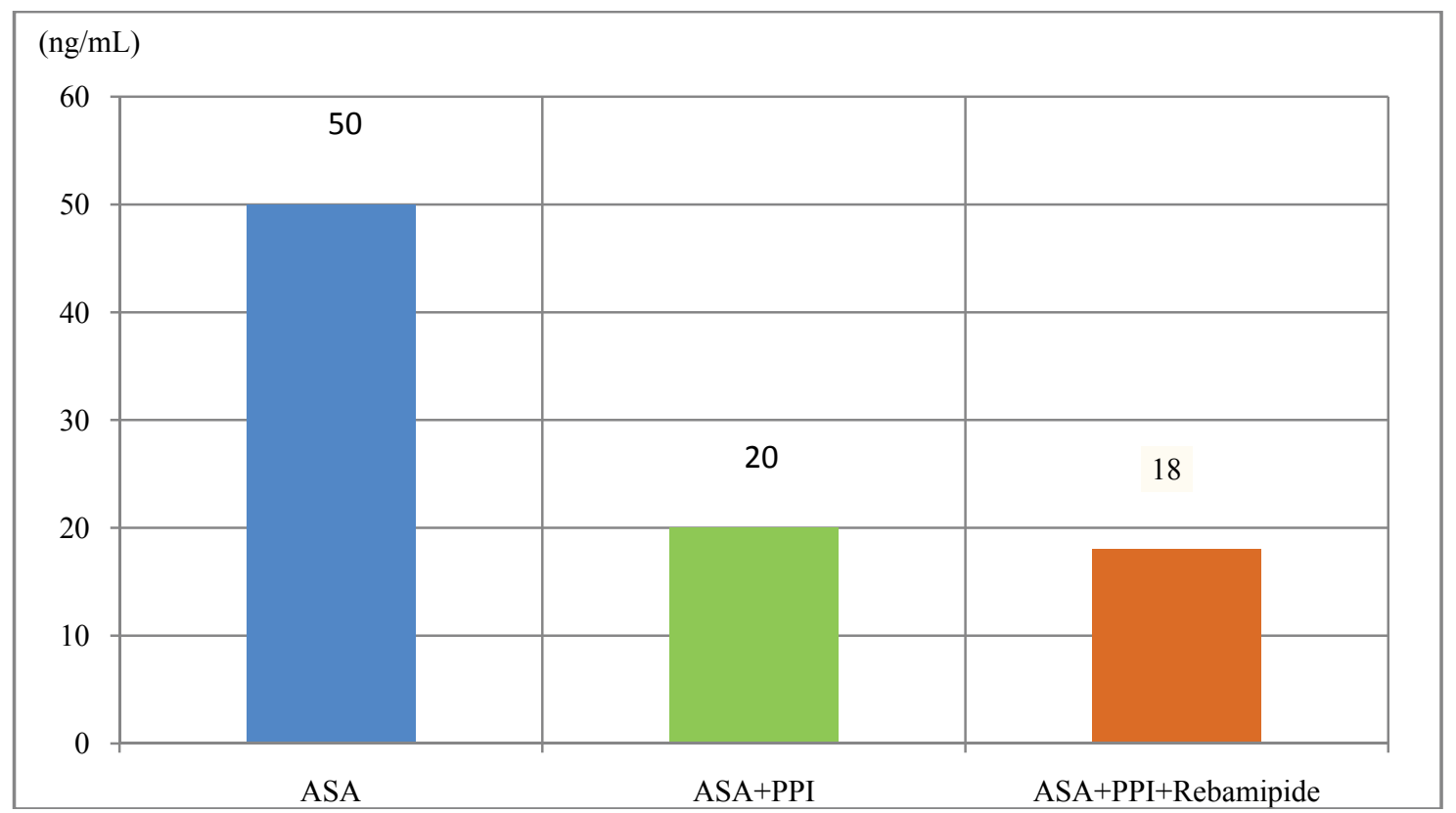

Fig. 1 LTB4 levels in patients with NSAIDs-gastropathy after ASA, pantoprazole and rebamipide treatment. 


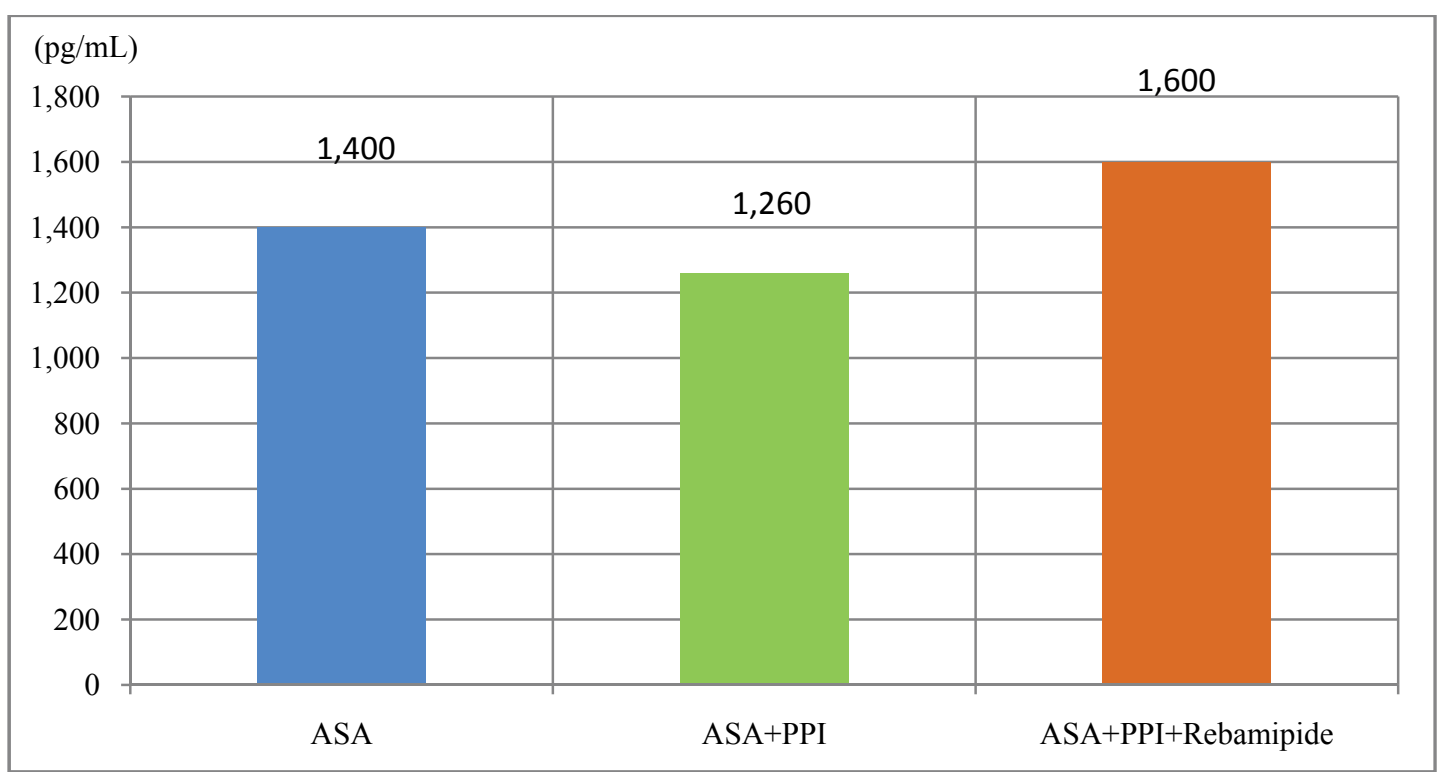

Fig. 2 PGE2 levels in patients with NSAIDs-gastropathy after ASA, pantoprazole and rebamipide treatment.

\section{Discussion}

Primary prevention of gastropathy and gastrointestinal bleeding is a strategic task in the course of ASA and other NSAIDs antiplatelet therapy. PPIs are the most effective class of drugs for the NSAIDs-induced stomach and duodenal ulcers and erosions relapse prevention. Among the PPI, pantoprazole has the lowest affinity for cytochrome P450. These data give reason to recommend pantoprazole for the prevention and treatment of the gastrointestinal tract complications during ASA therapy. However, according to the results, the choice method of gastroduodenal erosive and ulcerative lesions treatment is not only PPI, but rebamipide.

It has been demonstrated many ulcers and erosive defects in the group of patients, who were treated by aspirin on endoscopy research. However, this number significantly decreased in patients after pantoprazole treatment. In the group of patients, which were treated by rebamipide, ulcers weren't found and the number of erosive defects was significantly less in comparison to the other two groups.

Consideration of the LTB4 and PgE2 values relationship showed that under the influence of a pantoprazole and rebamipide combination in patients with NSAID-gastropathies, LTB4 levels mainly decreased and PGE2 content had raised. With the assignment only pantoprazole, changes were recorded in LTB4 level, while PGE2 protective factor decreased a little.

Thus, rebamipide in combination with pantoprazole not only reduces the factors of aggression to the gastroduodenal mucosa, but also improve its protective properties in patients with NSAIDs-gastropaty.

\section{Conclusions}

Pantoprazole and rebamipide combination provides efficacy in reducing the number of gastric ulcers, gastric erosions and duodenal ulcers and its healing in patients with NSAIDs gastropathy.

Pantoprazole and rebamipide therapy in patients with NSAIDs gastropathy led to the significant LTB4 decreasing with PGE2 content increasing simultaneously.

\section{References}

[1] Lanas, A. 2011. "Low Doses of Acetylsalicylic Acid Increase Risk of Gastrointestinal Bleeding in a Meta-analysis." Clinical Gastroenterology and Hepatology 9: 762-8.

[2] Becker, J., Domschke, W., and Pohle, T. 2004. "Current Approaches to Prevent NSAID-Induced Gastropathy-COX Selectivity and Beyond.” British Journal of Clinical Pharmacology 58 (6): 587-600. 
[3] Rao, P., and Knaus, E. 2008. "Evolution of Nonsteroidal Anti-inflammatory Drugs (NSAIDs): Cyclooxygenase (COX) Inhibition and Beyond." Journal of Pharmacy \& Pharmaceutical Sciences 11 (2): 81-110

[4] Wallace, J. L. 2008. "Prostaglandins, NSAIDs, and Gastric Mucosal Protection: Why Doesn't the Stomach Digest Itself?.” Physiological Reviews 88 (4):1547-65.

[5] Kang, G. M., Kim, N., and Lee, B. H. 2011. "Risk Factors for Peptic Ulcer Bleeding in Terms of Helicobacter Pylori, NSAIDs and Antiplatelet Agents." Scandinavian Journal of Gastroenterology 46: 1295-1301

[6] Fornai, M., Natale, G., Colucci, R., Tuccori, M., and Carazzina, G. 2005. "Mechanisms of Protection by Pantoprazole against NSAIDs-Induced Gastric Mucosal Damage." Naunyn-Schmiedeberg's Archives of
Pharmacology 372 (1): 79-87.

[7] Genta, R. M. 2003. "Review Article: The Role of Rebamipide in the Management of Inflammatory Disease of the Gastrointestinal Tract." Alimentary Pharmacology \& Therapeutics 18 (Suppl. 1): 8-13.

[8] Zhang, S., Qing, Q., Bai, Y., Mao, H., and Zhu, W. 2013. "Rebamipide Helps Defend against Nonsteroidal Anti-inflammatory Drugs Induced Gastroenteropathy: A Systematic Review and Meta-analysis." Digestive Diseases and Sciences 58 (7): 1991-2000.

[9] Yamao, J., Kikuchi, E., Matsumoto, M., Nakayama, M., and Ann, T. 2006. "Assessing the Efficacy of Famotidine and Rebamipide in the Treatment of Gastric Mucosal Lesions in Patients Receiving Long-Term NSAID Therapy." Journal of Gastroenterology 41 (12): 1178-85. 